\title{
Typisierung der Dienstvertretung im Zivilgesetzbuch der Volksrepublik China
}

\author{
CHI Ying
}

Mit $₫ 170$ des Zivilgesetzbuchs der Volksrepublik China $(\mathrm{ZGB})^{1}$ hat der chinesische Gesetzgeber das Rechtsinstitut der Dienstvertretung gesetzlich geregelt. Nach der h.L. wird die Handelsvertretung des zivilrechtlichen Systems mit der Kodifizierung der Dienstvertretung in das ZGB aufgenommen, welches ein Kennzeichen dafür ist, dass das ZGB das Zivilrecht und Handelsrecht einheitlich regelt. ${ }^{2}$ Jedoch bezeichnet $\$ 170$ ZGB einerseits zu viele und andererseits zu wenige handelsrechtliche Merkmale, weil der Gesetzgeber die Besonderheiten der Handelsregelungen nicht genug berücksichtigt hat. ${ }^{3}$ Es wird deshalb vorgeschlagen, die Dienstvertretung solle angesichts ihres handelsrechtlichen Charakters durch die Allgemeinen Grundsätze des Handelsrechts geregelt werden. ${ }^{4}$ Wegen der Langwierigkeit und Ungewissheit des Gesetzgebungsverfahrens bei der Kodifizierung der Allgemeinen Grundsätze des Handelsrechts ist dies jedoch unrealistisch. Stattdessen sollte eine Konkretisierung und Typisierung des $₫ 170$ ZGB nach den Prinzipien des Handelsrechts erfolgen, indem die Tatbestandmerkmale und Rechtsfolgen des $\$ 170$ ZGB klarer dargestellt werden.

1 中华人民共和国民法典 v. 28.05.2020, Amtsblatt des Ständigen Ausschusses des Nationalen Volkskongresses (中华人民共和国全国人民代表大会常务委员会公报) 2020, Nr.1, S. 1 ff.; deutsche Übersetzung in: Yijie DING/Nils Klages/Peter Leibküchler/Knut Benjamin Pißler, German Journal of Chinese Law (ZChinR), Vol. 27, No. 3 \& 4, S. 207-417.

2 YANG Qiuyu (杨秋宇), Integration von Zivil- und Handelsrecht: Konstitutionslogik und die normative Konstitution der Dienstvertretung (融贯民商：职务代理的 构造逻辑与规范表达), Science of Law (Journal of Northwest University of Political Science and Law) (法律科学 (西北政法大学学报) 2020 Nr. 1, S. 101; NIE Weifeng (聂卫锋), Normativer Ansatz und gesetzliche Formulierung der Dienstvertretung Kommentierung zu Artikel 170 der "Allgemeinen Grundsätze des Zivilrechts" (职权代理的规范理路与法律表达——<民法总则>第 170 条评析), Northern Legal Science (北方法学) 2018, Nr. 2, S. 63.

3 YANG Qiuyu (Fn. 2), S. 106.

4 ZHANG Gu (张谷), Reflexion über die heutige Kodifikation des Zivilrechts (对当 前民法典编纂的反思), ECUPL Journal (华东政法大学学报) 2016 Nr. 1, S. 5; YANG Qiuyu (Fn. 2), S. 108 ff. 
Es wird vertreten, dass die $\$ \mathbb{S} 48 \mathrm{ff}$. des deutschen Handelsgesetzbuchs $(\mathrm{HGB})^{5}$ bei der Auslegung des $₫ 170$ ZGB als Vorbild gelten sollen und die Dienstvertretung in China anhand der deutschen Prokura und Handlungsvollmacht zu typisieren sei. ${ }^{6}$ Dieser Ansicht ist sich anzuschließen. Auf dieser Grundlage werden im Folgenden die Einordnung, der Begriff und die Tatbestandsmerkmale der Dienstvertretung, die Erteilung, der Umfang und die Grenzen der Dienstvertretungsmacht sowie die Rechtsfolgen der Vertretung ohne Vertretungsmacht und der Überschreitung der Grenzen im Innenverhältnis rechtsvergleichend unter Berücksichtigung der chinesischen Literatur und Rechtsprechung einerseits und der Gesetzgebung, Rechtsprechung und Dogmatik des deutschen Handelsrechts andererseits analysiert und kommentiert. Letztlich sollen die Schnelligkeit und Sicherheit des Handelsverkehrs gefördert und gleichzeitig die Interessen des Vertretenen gewährleistet werden. Auch soll diese Arbeit dem chinesischen Obersten Volksgericht eine theoretische Grundlage und eine rechtsvergleichende Ansicht für die mögliche Auslegung des $\$ 170$ ZGB durch die Rechtsprechung bieten.

\section{Begriff, Einordnung und Tatbestandsmerkmale der Dienstvertretung}

\section{Einordnung der Dienstvertretung}

Die Dienstvertretung wird in der chinesischen Literatur entweder als gesetzliche Vertretung, rechtsgeschäftliche Vertretung oder gemischte Vertretung eingeordnet.

5 Handelsgesetzbuch in der im Bundesgesetzblatt Teil III, Gliederungsnummer 4100-1, veröffentlichten bereinigten Fassung, das zuletzt durch Artikel 3 des Gesetzes vom 12. 12. 2019 (BGBl. I S. 2637) geändert worden ist.

6 ZHANG Gu (张谷), Zum Verständnis und zur Anwendung der "Allgemeinen Grundsätze des Zivilrechts" aus Sicht des Verhältnisses zwischen Zivilrecht und Handelsrecht (从民商关系角度谈《民法总则》的理解与适用), China Review of Administration and Justice (中国应用法学) 2017 Nr. 4, S. 156. 


\section{a) Gesetzliche Vertretung}

Die diese Ansicht vertretenden Autoren sind der Meinung, die Dienstvertretung sei im Kapitel der juristischen Personen im ZGB zu regeln. ${ }^{7}$ Dieser Ansicht ist nicht beizutreten. Obwohl $\$ 43$ der Allgemeinen Grundsätze des Zivilrechts (AGZR) ${ }^{8}$ keinen Unterschied zwischen gesetzlichen Repräsentanten und Vertretungen durch sonstige Mitarbeiter macht, hat der Gesetzgeber des ZGB immerhin den gesetzlichen Repräsentanten im Kapitel der juristischen Personen und die Dienstvertretung im Kapitel über die Stellvertretung separat geregelt.

Nach $₫ 61$ Abs. 1 ZGB kann der gesetzliche Repräsentant der juristischen Person im Namen von juristischen Personen rechtsgeschäftlich handeln. Folglich handelt es sich um eine gesetzliche Vertretungsmacht des gesetzlichen Repräsentanten. ${ }^{9}$ Darüber hinaus bestimmt $\$ 13$ Gesellschaftsgesetz (GG) ${ }^{10}$ dass der Vorstandsvorsitzende, Geschäftsführer oder Manager als gesetzlicher Repräsentant berufen werden kann. In diesem Sinne ist der gesetzliche Repräsentant vergleichbar mit dem gesetzlichen Vertreter. Hingegen ist nach $\$ 170$ ZGB weder die Dienstvertretungsmacht eine gesetzliche Vertretungsmacht noch wird der Dienstvertreter von Gesetzes wegen hierzu berufen, weshalb die Dienstvertretung nicht als gesetzliche Vertretung einzuordnen ist. Wahrscheinlich ist dies auch der Grund, warum der Dienstvertreter gesondert von dem gesetzlichen Repräsentanten geregelt wurde.

7 XIE Hongfei (谢鸿飞), Rechtsgrundlage und wichtige Rechtsinstitute der Gesetzgebung über die Stellvertretung (代理部分立法的基本理念和重要制度), ECUPL Journal (华东政法大学学报) 2016, Nr. 5, S. 66.

8 中华人民共和国民法通则 v. 12.4.1986, Gesetzsammlung (法规汇编) 1986, S. 1 ff.; deutsche Übersetzung in: Frank Münzel (Hrsg.), Chinas Recht III. 7, 12.4.86/1. Es ist am 1.1.2021 außer Kraft getreten.

9 LIU Junhai (刘俊海), Modernes Gesellschaftsrecht (现代公司法), 3. Auflage, Beijing 2015, S. 612.

10 中华人民共和国公司法 v. 27.10.2005, revidiert am 28.12.2013, nochmals revidiert am 26.10.2018, Amtsblatt des Ständigen Ausschusses des Nationalen Volkskongresses (中华人民共和国全国人民代表大会常务委员会公报) 2018，Nr.6， S. $789 \mathrm{ff}$; englische Übersetzung in: <www.lawinfochina.com> eingesehen am 6.6.2021. 


\section{b) Gemischte Vertretung}

Nach dieser Theorie ist die Dienstvertretung sowohl als gesetzliche als auch als rechtsgeschäftliche Vertretungsmacht einzuordnen, ${ }^{11}$ weil der Umfang der Dienstvertretungsmacht vom Gesetz bestimmt und die Vollmacht vom Vollmachtgeber nach seinem Willen erteilt wird. ${ }^{12}$ Dieser Ansicht ist nicht zuzustimmen. Die gesetzliche Bestimmung des Umfangs der Dienstvertretungsmacht dient nur dazu, den Umfang der Dienstvertretungsmacht bekanntzumachen, damit der Vertragspartner sich ihrer nicht rückzuversichern braucht. Die Bevollmächtigung ist eine Willenserklärung des Vollmachtgebers aufgrund der Privatautonomie. Darüber hinaus kann der Vollmachtgeber den Umfang der Dienstvertretung nach den gesetzlichen Regelungen rechtsgeschäftlich erweitern.

\section{c) Rechtsgeschäftliche Vertretung}

Nach der h.M. ist die Dienstvertretung eine besondere Art der rechtsgeschäftlichen Vertretung (Vollmacht), obwohl die Dienstvertretung einige von der Vollmacht abweichende Merkmale aufweist. ${ }^{13}$ Diese Ansicht ist überzeugend. Denn das ZGB hat die Dienstvertretung unter dem Titel der rechtsgeschäftlichen Vertretung aufgenommen, wobei sich die Besonderheiten der Dienstvertretung im Vergleich zur Vollmacht in zweierlei Hinsicht zeigen: Zum einen ist der Dienstvertreter gleichzeitig auch der Mitarbeiter des Vertretenen und zum anderen wird der Umfang der Dienstvertretung vom Gesetz bestimmt.

11 ZHAO Wanyi (赵万一), Unternehmensmanager und Prokura (公司经理与经理 权), Beijing 2013, S. 61; YANG Qiuyu (Fn. 2), S. 102.

12 YANG Qiuyu (Fn. 2), S. 102.

13 WANG Liming (王利明), Untersuchung zu den allgemeinen Grundsätzen des Zivilrechts (民法总则研究)，3. Auflage，Beijing 2018，S. 613; LI Shishi (李适 时), Auslegung der Allgemeinen Grundsätze des Zivilrechts der Volksrepublik China (中华人民共和国民法总则释义), Beijing 2017, S. 528; MA Junju (马俊 驹)/YU Yanman (余延满), Grundsätze des Zivilrechts (民法原论), Auflage 4, Beijing 2010, S. 224-225; YANG Lixin (杨立新), Geschichten und Probleme hinter den Paragraphen (条文背后的故事与难题), Beijing 2017, S. 431; ZHANG Xinbao (张新宝), Auslegung der Allgemeinen Grundsätze des “Zivilrechts der Volksrepublik China” (<中华人民共和国民法总则>释义), Beijing 2017, S. 368; YIN Fei (尹 飞), Quelle der Vollmacht aus der Sicht der Systematisierung (体系化视角下的意 定代理权来源) , Faxue Yanjiu (法学研究) 2016, Nr. 6, S. 51; ZHANG Gu (Fn. 6), S. 155; YANG Qiuyu (Fn. 2), S. 102. 


\section{d) Handelsvertretung}

Vor dem Erlass des ZGB wurde vertreten, dass die AGZR zwar die Dienstvertretung des gesetzlichen Repräsentanten und des sonstigen Mitarbeiters separat geregelt hätten, aber keine Regelung zur Handelsvertretung wie die Prokura oder Handlungsvollmacht beinhalteten. ${ }^{14}$ Diese Ansicht ist nicht überzeugend. Wäre die Dienstvertretung keine Handelsvertretung, dann wäre sie nicht gesondert geregelt worden, weil die zivilrechtliche Stellvertretung hierfür ausreicht. Vereinzelt wird darauf hingewiesen, dass sie durch die Rechtsinstitute der rechtsgeschäftlichen Vertretung und Anscheinsvollmacht gedeckt sei. ${ }^{15}$ Dagegen ist der h.M. zuzustimmen, dass die Dienstvertretung eine Art der Handelsvertretung darstellt und mit ihrer Regelung im ZGB die Handelsvertretung ein Teil des Stellvertretungsrechts geworden ist. ${ }^{16}$ Insofern hat die Kodifizierung der Dienstvertretung deutlich gemacht, dass das ZGB zum Teil auch handelsrechtliche Rechtsinstitute regelt.

In Wirklichkeit gibt es zwei Arten der Handelsvertretung, nämlich die Dienstvertretung und die sonstige von selbständigen gewerbetreibenden Handelsvertretern unternommene Handelsvertretung (Handelsvertretung nicht kraft des Amtes), wovon das ZGB aber nur erstere geregelt hat. Die Dienstvertretung ähnelt der Prokura und Handlungsvollmacht ( $\$ 48 \mathrm{ff}$. HGB), während die Handelsvertretung nicht kraft des Amtes Ähnlichkeiten zur Handelsvertretung ( $\mathbb{S} 84 \mathrm{ff}$. HGB) im deutschen Recht aufweist.

Zusammenfassend ist die Dienstvertretung als eine Handelsvertretung einzuordnen und unterscheidet sich von dem gesetzlichen Repräsentanten und der rechtsgeschäftlichen Vertretung. Im Folgenden werden Begriff und Tatbestandsmerkmale der Dienstvertretung analysiert.

\section{Begriff und Tatbestandsmerkmale der Dienstvertretung}

Nach der h.L. entsteht die Dienstvertretung aufgrund des Arbeitsverhältnisses zwischen Vertreter und Dienstherrn, ohne dass separat eine Voll-

14 JIANG Daxing (蒋大兴)/WANG Shoujie (王首杰), Anpassung der Handelsvertretung in den allgemeinen Grundsätzen des Zivilrechts（论民法总则对商事代理的 调整), Social Sciences in Guangdong (广东社会科学) 2016 Nr. 1, S. 231.

15 NIE Weifeng (Fn. 2), S. 64.

16 RAN Keping (手克平), Zur Handelsvertretung und deren Systemstruktur (论商 事职务代理及其体系构造), Studies in Law and Business (法商研究) 2021, Nr. 1, S. 140. 
macht erteilt werden muss (Einheitstheorie). ${ }^{17}$ Dieser Theorie ist nicht zuzustimmen, weil sie keinen Unterschied zwischen Innenverhältnis und Vollmachtserteilung macht und der Wille des Vertretenen nicht berücksichtigt wird. Sie verstößt gegen das Prinzip der Privatautonomie, da der Vertretene sonst das von einem nicht bevollmächtigten Mitarbeiter getätigte Rechtsgeschäft für oder gegen sich gelten lassen müsste.

Dagegen ist der Trennungstheorie ${ }^{18}$ zuzustimmen, wonach nur eine separate Bevollmächtigung den Mitarbeiter berechtigt, im Namen des Vertretenen als Dienstvertreter aufzutreten. Aus dem Arbeitsvertrag zwischen dem Vertretenen und dem Mitarbeiter hingegen ist keine Vertretungsmacht abzuleiten, da dieser bloß die Stellung des Mitarbeiters bestimmt. In der Rechtsprechung haben einige Gerichte hierzu entschieden, dass die Dienstvertretung ohne Bevollmächtigung eine Vertretung ohne Vertretungsmacht darstellt mit der Folge, dass eine separate Vollmachtserteilung für die Dienstvertretung unverzichtbar ist. ${ }^{19}$

Der Dienstvertreter muss grundsätzlich im Namen des Vertretenen handeln. Jedoch gilt ein nicht im Namen des Vertretenen geschlossenes Rechtsgeschäft auch dann als Dienstvertretung, wenn die betreffende Handlung im Geschäftsraum des Vertretenen stattgefunden hat und offensichtlich keine Privatangelegenheit ist. ${ }^{20}$ Wenn der Dienstvertreter irrtümlich nicht im Namen des Vertretenen handelt, ist dieser Irrtum unter Berücksichtigung der Verkehrssicherheit nicht anfechtbar. Hierzu wird rechtsvergleichend auf die Gesetzesregelung des $₫ 164$ Abs. 2 des Bürgerlichen Gesetzbuchs (BGB) ${ }^{21}$ zurückgegriffen, die auch für die Prokura gilt. ${ }^{22}$ In der chinesischen Rechtsprechung haben einige Gerichte etwa eine

17 JIANG Ping (江平)/ZHANG Peilin (张佩霖), Lehrbuch des Zivilrechts, Beijing 1986, S. 95; WANG Liming (Fn. 13), S. 613; LI Shishi (Fn. 13), S. 527; WANG Xiaohua (汪晓华), Gedankenslogik der gerichtlichen Entscheidung zur Dienstvertretung des Zivilrechts (民事职务行为司法判定的逻辑理路), Hebei Law Science (河北法学) 2019, Nr. 3, S. 118; RAN Keping (Fn. 16), S. 141.

18 XU Shencheng (徐深澄), Systematische Auslegung der Dienstvertretung der „Allgemeinen Grundsätze des Zivilrechts“ (<民法总则>职务代理规则的体系化阐释), The Jurist (法学家) 2019, Nr. 2, S. 100.

19 Zivilurteil des Mittleren Volksgerichts der Stadt Nanchong, Provinz Sichuan (2018) Chuan 13 Min Zhong 2436; Zivilurteil des Mittleren Volksgerichts der Stadt Liaocheng, Provinz Shandong (2018) Lu 15 Min Zhong 3272.

20 Peter Krebs, in: Ingo Drescher/Holger Fleischer/Karsten Schmidt (Hrsg.), Münchener Kommentar zum HGB, 5. Auflage, München 2021, vor $₫ 48$ Rn. 48.

21 Bürgerliches Gesetzbuch in der Fassung der Bekanntmachung vom 2. Januar 2002 (BGBl. I S. 42, 2909; 2003 I S. 738), das zuletzt durch Artikel 1 des Gesetzes vom 4. Mai 2021 (BGBl. I S. 882) geändert worden ist.

22 Klaus Hofmann, Der Prokurist, 7. Auflage, Heidelberg 1996, S. 101. 
Dienstvertretung abgelehnt, wenn der Dienstvertreter die Zahlung des Kaufpreises nicht im Namen des Vertretenen angenommen hat. ${ }^{23}$ Solche Entscheidungen sind nicht überzeugend, da dadurch die Verkehrssicherheit stark gefährdet wird. Die Dienstvertretungsmacht soll auch die Annahme des Kaufpreises decken, ohne dass der Vertragspartner zu bestätigen braucht, dass der Dienstvertreter im Namen des Vertretenen den Kaufpreis annimmt. Für die Dienstvertretung muss es bereits ausreichen, wenn der Dienstvertreter im Namen des Vertretenen den Vertrag schließt.

\section{Typen und Erteilung der Dienstvertretungsmacht}

\section{Typen der Dienstvertretungsmacht}

Die Typisierung der Dienstvertretungsmacht wurde als eine allgemeine Gesetzgebungstechnik in vielen Ländern mit kontinentaleuropäischer Rechtstradition vollzogen. Die Typisierung der Dienstvertretungsmacht ist nicht nur eine Anforderung der Privatautonomie, sondern auch eine wichtige Maßnahme zur Förderung der Verkehrssicherheit. ${ }^{24}$ Mit der gesetzlichen Typisierung der Dienstvertretungsmacht braucht der Vertragspartner den Umfang der Dienstvertretungsmacht nicht umfassend zu überprüfen. ${ }^{25}$ Darüber hinaus dient die Typisierung auch als eine Beschränkung der Dienstvertretungsmacht, die die Interessen des Vertretenen schützt. Die Prokura und die Handlungsvollmacht sind zwei wichtige Typen der Dienstvertretungsmacht im deutschen HGB. Da die Bestimmungen des $\$ 170$ ZGB sehr allgemein formuliert und die Sicherheit und Schnelligkeit des Handelsgeschäfts hierdurch nicht gewährleisten werden können, ist es erforderlich, die Dienstvertretung in China parallel wie im deutschen HGB in Prokura und Handlungsvollmacht zu typisieren.

23 Zivilurteil des Mittleren Volksgerichts der Stadt Deyang, Provinz Sichuan (2020) Chuan 06 Min Zai 13; Zivilurteil des Mittleren Volksgerichts der Stadt Guangan, Provinz Sichuan (2020) Chuan 16 Min Zhong 406.

24 XU Shencheng (Fn. 18), S. $102 \mathrm{ff}$.

25 MüKoHGB/Krebs, 5. Auflage 2021, vor $₫ 48$ Rn. 33; CHEN Ziqiang (陈自强), Zwischen Vollmacht und Prokura - Einheit und Trennung von Zivil- und Handelsrecht (代理权与经理权之间——民商合一与民商分立), Beijing 2008, S. 131. 


\section{a) Prokurist}

Der Manager im Sinne des GG ist kein Prokurist im Handelsverkehr. Nach \49 GG hat der Manager nur die Befugnis zur Ausführung der inneren Aufgaben der Gesellschaft und keine Vertretungsmacht nach außen. ${ }^{26}$ Nur mit einer separaten Bevollmächtigung vom Vorstand nach $\$ 49$ Abs. 8 GG kann der Manager die Gesellschaft nach außen vertreten. ${ }^{27}$ Darüber hinaus kann der Manager nach $\$ 13$ GG auch der gesetzliche Repräsentant der Gesellschaft sein, dessen Vertretungsmacht vom Gesetz bestimmt wird. Wenn der Manager zugleich der gesetzliche Repräsentant ist, kann er zwar die Gesellschaft nach außen vertreten, aber dies nur als gesetzlicher Repräsentant (wie die Vorstandsvertretung im deutschen Recht) und nicht als Prokurist. Um Prokurist zu sein, muss ihm vielmehr eine separate Prokura erteilt worden sein.

Da der Begriff des Prokuristen im chinesischen Recht nicht eindeutig ist, ist es notwendig, diesen Begriff in Zusammenhang mit dem deutschen Recht zu verdeutlichen. Der Prokurist im Sinne des deutschen HGB ist das zweite Ich des Geschäftsinhabers und kann Geschäfte jedweder Art schließen; kurzum ersetzt er den Geschäftsherrn im geschäftlichen Verkehr und im Rechtsverkehr. ${ }^{28}$ Danach ist die Prokura eine vom Handelsgewerbe erteilte umfangreiche Vollmacht im Handelsverkehr. Mit der Typisierung der Dienstvertretung ist der Begriff des Prokuristen auch im chinesischen Recht zu übernehmen.

\section{b) Handlungsvollmacht}

Manche Autoren sind der Auffassung, dass die Handlungsvollmacht in der chinesischen Rechtsprechung abhängig von der Arbeitsstelle in Vertriebs-

26 RAN Keping (Fn. 16), S. 140.

27 GAN Peizhong (甘培忠), Untersuchung der Stellvertretung im Gesellschaftsrecht (公司代理制度论略), China Legal Science (中国法学) 1997, Nr. 6, S. 73; FAN Jian (范建)/JIANG Daxing (蒋大兴), Vergleichende Untersuchung zu den Rechtsfragen der Prokura im Gesellschaftsrecht (公司经理权法律问题比较研究), Journal of Nanjing University (Philosophy, Humanities and Social Sciences) (南京大 学学报/哲学.人文.社会科学) 1998, Nr.3, S. 147.

28 Reimar Spitzbarth, Vollmachten im modernen Management: Handlungsvollmacht, Prokura, Generalvollmacht, Berlin 1970, S. 57; Klaus Hofmann (Fn. 22), S. 13. 
manager, Projektmanager etc. zu unterteilen ist. ${ }^{29}$ Jedoch gibt es in der chinesischen Literatur kaum Erläuterungen zum Begriff der Handlungsvollmacht, sodass hierzu $₫ 54$ HGB herangezogen werden muss: Danach ist die Handlungsvollmacht eine Vollmacht, die dem Bevollmächtigten zum Betrieb eines Handelsgewerbes oder zur Vornahme einer bestimmten zu einem Handelsgewerbe gehörigen Art von Geschäften oder zur Vornahme einzelner zu einem Handelsgewerbe gehöriger Geschäfte bevollmächtigt, die der Betrieb eines derartigen Handelsgewerbes oder die Vornahme derartiger Geschäfte gewöhnlich mit sich bringt. Im Vergleich zur Prokura beschränkt sich die Handlungsvollmacht auf gewöhnliche Geschäfte des Handelsgewerbes.

\section{Erteilung der Dienstvertretungsmacht}

\section{a) Geschäftsherr}

Nach $₫ 170$ ZGB gehören zum Kreis des Geschäftsherrn juristische Personen oder Organisationen ohne Rechtspersönlichkeit. Hinsichtlich juristischer Personen ist zwischen gewinnorientierten juristischen Personen $(\mathbb{S} 76 \mathrm{ZGB})$, nicht gewinnorientierten juristischen Personen $(\mathbb{S} 87$ ZGB) und besonderen juristischen Personen $(\$ 96$ ZGB) zu unterscheiden. Organisationen ohne Rechtspersönlichkeit sind unter anderem Einzelunternehmen, Partnerschaftsunternehmen sowie Einrichtungen für professionelle Dienstleistungen ohne Rechtspersönlichkeit ( $\$ 102$ ZGB). Daher fallen nicht nur Handelsgewerbe, sondern auch solche, die kein Handelsgewerbe sind, unter $₫ 170$ ZGB. Es wird kritisiert, dass der Begriff des Geschäftsherrn in $₫ 170 \mathrm{ZGB}$ zu weitgehend und deshalb einschränkend auszulegen sei, sodass nur ein Handelsgewerbe eine Dienstvertretungsmacht erteilen dürfe. ${ }^{30}$ Dieser Ansicht ist beizutreten. Wie bereits gesehen stellt die Dienstvertretung eine Art der Handelsvertretung dar. Somit sollte der Geschäftsherr des Dienstvertreters ein Kaufmann sein, da auch rechtsvergleichend im deutschen HGB der Geschäftsherr der Prokura und Handlungsvollmacht ein Kaufmann ist. Meiner Ansicht nach können nur ge-

29 RAN Keping (苒克平)/QU Yanni (罌燕妮), Untersuchung der Handelsvertretung und ihre Verbesserung - Eine Analyse von Artikel 170 der „Allgemeinen Grundsätze des Zivilrechts“(论我国的商事职务代理制度及其完善—一兼析<民 法总则>第 170 条), Journal of Hubei University of Police (湖北警官学院学报) 2019, Nr. 4, S. 73.

30 ZHANG Gu (Fn. 4), S. 155. 
winnorientierte juristische Personen, juristische Personen der städtischen und ländlichen genossenschaftlichen Wirtschaftsorganisation und juristische Personen des ländlichen Kollektivwirtschaftsverbandes als besondere juristischen Personen, sowie Einzelunternehmen und Partnerschaftsunternehmen als Organisationen ohne Rechtspersönlichkeit im chinesischen Recht eine Dienstvertretungsmacht erteilen. Dagegen sind nicht gewinnorientierte juristische Personen (Institutionseinheiten, gesellschaftliche Körperschaften, Stiftungen und Einrichtungen für soziale Dienste), behördliche juristische Personen, juristische Personen der Basisorganisationen der Selbstverwaltung sowie Einrichtungen für professionelle Dienstleistungen als Organisation ohne Rechtspersönlichkeit keine Kaufleute. Für solche nicht-kaufmännischen juristischen Personen sind der gesetzliche Repräsentant und die zivilrechtliche Vollmacht als ausreichend anzusehen.

\section{b) Bevollmächtigte}

Wie oben erläutert gelten Dienstvertretung und Handelsvertretung nicht kraft des Amtes als zwei Arten der Handelsvertretung. Für eine Dienstvertretung muss zwischen Bevollmächtigtem und dem Handelsgewerbe ein Arbeitsverhältnis bestehen. Es ist in der Literatur auch allgemein anerkannt, dass der Bevollmächtigte bei der Dienstvertretung vom Geschäftsherrn beschäftigt sein sollte, entweder als regulärer Mitarbeiter oder als Personal der Arbeitsabfertigung. ${ }^{31}$ Dagegen sind Rechtsanwälte, Makler oder Sublieferanten, die nicht in einem Arbeitsverhältnis mit dem Geschäftsherrn stehen, keine Dienstvertreter, weil sie keine Arbeitsstelle im Handelsgewebe innehaben und keinen Dienst ausführen. In der Rechtsprechung ist ebenfalls anerkannt, dass das Arbeitsverhältnis zwischen dem Dienstvertreter und dem Geschäftsherrn eines der Tatbestandsmerkmale der Dienstvertretung darstellt, ${ }^{32}$ auch wenn ein einzelnes Gericht geurteilt

31 YIN Fei (Fn. 13), S. 60; YANG Qiuyu (Fn. 2), S. 105; LI Shishi (Fn. 13), S. 527.

32 Zivilurteil des Mittleren Volksgerichts der Stadt Qingdao, Provinz Shandong (2020) Lu 02 Min Zhong 9352; Zivilurteil des Mittleren Volksgerichts der Stadt Deyang, Provinz Sichuan (2020) Chuan 06 Min Zai 13; Zivilurteil des Mittleren Volksgerichts der Stadt Qingdao, Provinz Shandong (2020) Lu 02 Min Zhong 6426; Zivilurteil des Mittleren Volksgerichts der Stadt Jiujiang, Provinz Jiangxi (2020) Gan 04 Min Zhong 1090. 
hat, dass der Sublieferant angesichts seiner engen Beziehung mit der Gesellschaft als Dienstvertreter der Gesellschaft gilt. ${ }^{33}$

Angesichts des nötigen persönlichen Vertrauens zwischen dem Geschäftsherrn und Bevollmächtigten kann nur eine natürliche Person mit voller Handlungsfähigkeit Dienstvertreter sein. Daher kann ein Handelsgewerbe selbst kein Dienstvertreter sein, weil es an dem nötigen persönlichen Vertrauen aufgrund häufiger Änderung des gesetzlichen Repräsentanten oder Geschäftsführers des Handelsgewerbes fehlt. Um für klare Rechtsverhältnisse zu sorgen, kann der gesetzliche Repräsentant der juristischen Person nach der h.M. auch nicht gleichzeitig Dienstvertreter sein. ${ }^{34}$ Dagegen kann der Geschäftsführer des Handelsgewebes ohne Rechtspersönlichkeit mangels einer gesetzlichen Regelung Dienstvertreter i. S. v. $\$ 170$ ZGB sein, ${ }^{35}$ weil $\ 61$ ZGB sich nur auf den gesetzlichen Repräsentanten einer juristischen Person bezieht.

\section{c) Erteilung der Dienstvertretungsmacht}

\section{(1) Erteilung der Prokura}

Die Erteilung der Prokura ist eine empfangsbedürftige einseitige Willenserklärung. Die Prokura kann nur durch den Geschäftsinhaber, dessen gesetzlichen Vertreter oder den gesetzlichen Repräsentanten der juristischen Person sowie durch den Geschäftsinhaber oder dessen gesetzlichen Vertreter von Organisationen ohne Rechtspersönlichkeit erteilt werden. Der Prokurist oder Handlungsbevollmächtigte ist selbst nicht in der Lage, eine weitere Prokura zu erteilen. Die Prokura kann nur ausdrücklich erteilt werden, weil der Umfang der Prokura sehr weit und für den Vollmachtgeber von großer Bedeutung ist. ${ }^{36}$ Darüber hinaus sind Duldungsvollmacht oder Anscheinsvollmacht bei der Prokura im Prinzip nicht anzuerkennen.

33 Zivilurteil des Ersten Mittleren Volksgerichts der Stadt Shanghai (2020) Hu 13 Min Zhong 9270.

34 WANG Liming (Fn. 13), S. 641; WANG Xiaohua (Fn. 17), S. 117; NIE Weifeng (Fn. 2), S. 60.

35 CHEN Su (陈廷生), Kommentar zu den Allgemeinen Grundsätzen des Zivilrechts, Beijing 2017, S. 1207; NIE Weifeng (Fn. 2), S. 61.

36 XU Shencheng (Fn. 18), S. 101; CHEN Ziqiang (Fn. 25), S. 125. 
Es wird in der chinesischen Literatur vorgeschlagen, dass die Erteilung der Prokura wie im deutschen Recht ${ }^{37}$ in die Geschäftslizenz (ähnlich wie das Handelsregister im deutschen Recht) eingetragen werden sollte, um Rechtsklarheit zu gewährleisten und Verwirrung und Missverständnisse bezüglich der Prokura zu vermeiden. ${ }^{38}$ Dieser Ansicht ist sich anzuschließen, da eine Eintragung der Prokura in die Geschäftslizenz und ihre Veröffentlichung auf einer Plattform zur Offenlegung von Unternehmensinformationen der öffentlichen Bekanntmachung der Prokura und dadurch der Verkehrssicherheit dienen würde.

Darüber hinaus sollte die Prokura wie im deutschen Recht ${ }^{39}$ als Gesamtprokura erteilt werden, um einen Missbrauch der Prokura zu vermeiden. In der deutschen Handelspraxis wird angesichts der kaum zu beschränkenden Prokura die Gesamtprokura ziemlich weit angewendet. ${ }^{40}$ Im chinesischen Recht ist die von $\mathbb{1 6 6}$ ZGB bestimmte Gesamtvollmacht entsprechend auch für die Prokura anzuwenden.

\section{(2) Erteilung der Handlungsvollmacht}

Bezüglich der Erteilung der Handlungsvollmacht kann das deutsche Recht als Vorbild herangezogen werden, weil eine Regelung über die Handlungsvollmacht im chinesischen Recht nicht vorhanden ist und kaum über die Erteilung der Handlungsvollmacht in der chinesischen Literatur diskutiert wird. Parallel wie im deutschen Recht sollte die Handlungsvollmacht nicht nur von dem Inhaber, dem gesetzlichen Vertreter oder dem gesetzlichen Repräsentanten des Unternehmens, sondern auch von einem Prokuristen erteilt werden, weil der Umfang der Prokura umfassend ist und der Prokurist dazu bevollmächtigt ist. Die Handlungsvollmacht sollte auch stillschweigend erteilt werden können, ${ }^{41}$ z. B. durch die Zuweisung

$37 \$ 53$ Abs. 1 S. 1 HGB bestimmt: “Die Erteilung der Prokura ist von dem Inhaber des Handelsgeschäfts zur Eintragung in das Handelsregister anzumelden."

38 JIANG Daxing/WANG Shoujie (Fn. 14), S.232; RAN Keping (Fn. 16), S. 142; RAN Keping/QU Yanni (Fn. 29), S. 73.

$39 \$ 48$ Abs. 2 HGB bestimmt: "Die Erteilung kann an mehrere Personen gemeinschaftlich erfolgen."

40 MüKoHGB/Krebs, 5. Auflage 2021, $\$ 48$ Rn. 71.

41 MüKoHGB, $₫ 54$ Rn. 35; XU Shencheng (Fn. 18), S. 101; RAN Keping (Fn. 16), S. 143. 
einer Aufgabe, für deren Ausführung normalerweise eine Handlungsvollmacht erforderlich ist. ${ }^{42}$

Anders als bei der Prokura ist eine Eintragung der Handlungsvollmacht nicht als erforderlich zu erachten, weil die Handlungsvollmacht nicht so umfangreich wie die Prokura ist und deshalb in das Recht des Geschäftsherrn nicht so tief eingreift wie die Prokura.

\section{Umfang der Dienstvertretungsmacht und deren Beschränkung}

Zugunsten der Verkehrssicherheit und zum Schutz des Bevollmächtigten sollte der Umfang der Dienstvertretung vom Gesetz bestimmt werden, um dem Vertragspartner Rückfragen hinsichtlich des Umfangs im Einzelfall zu ersparen. Gleichzeitig braucht dann auch der mit Dienstvertretungsmacht handelnde Bevollmächtigte nicht zu befürchten, dass er als Vertreter ohne Vertretungsmacht in Anspruch genommen wird. Dem wird jedoch durch die Regelung des $\$ 170$ ZGB nicht Genüge getan, da es sich um eine allgemeine und ungenaue Bestimmung handelt, die von Anfang an kaum praktische Bedeutung aufweist. Dem Vorschlag, die spezifischen und leicht zu identifizierenden Kriterien zur Beurteilung des Umfangs der Dienstvertretungsmacht unter Anwendung der Grundnormen zur Vollmacht und Anscheinsvollmacht durch eine Typisierung der Fälle in der Rechtsprechung herauszuarbeiten, ${ }^{43}$ ist nicht beizutreten, da dies sonst den Wert der Dienstvertretung als ein selbstständiges Rechtsinstitut ignoriert. Dagegen ist der h.M. zuzustimmen, wonach der Umfang der Dienstvertretungsmacht direkt vom Gesetz bestimmt werden sollte und der Dienstvertreter einzutragen sei. ${ }^{44}$ In dieser Hinsicht erweisen sich die Regelungen der $\$ \$ 48 \mathrm{ff}$. HGB als inspirierend, in der der Umfang der Prokura und der Handlungsvollmacht gesetzlich bestimmt und die Eintragung der Prokura vom Gesetz ausdrücklich geregelt sind. Im Folgenden wird der Umfang der Dienstvertretungsmacht im chinesischen Recht unter Berücksichtigung des deutschen Rechts bestimmt.

42 BGH NJW 2014, 2790 (2788); BGH NJW 2015, 2584 (2588).

43 NIE Weifeng (Fn. 2), S. 62 ff; YANG Qiuyu (Fn. 2), S. 101.

44 YIN Fei (Fn. 13), S. 69; ZHANG Gu (Fn. 6), S. 156. 


\section{Umfang der Dienstvertretungsmacht und deren gesetzliche Beschränkung}

\section{a) Die chinesische Rechtslage}

Über den Umfang der Dienstvertretungsmacht wird gestritten. Auf der einen Seite wird vorgeschlagen, der Umfang der Dienstvertretung sei nach der Arbeitsstellung des Dienstvertreters zu bestimmen. ${ }^{45}$ Auf der anderen Seite wird vertreten, der Umfang der Dienstvertretung sollte aufgrund der Typisierung des Dienstvertreters gesetzlich geregelt werden. ${ }^{46}$ Die erste Ansicht ist fragwürdig, da die Arbeitsstellung des Dienstvertreters vielfältig ist und es daher fast unmöglich ist, einheitliche Abgrenzungskriterien zu schaffen. Deshalb birgt die Bestimmung des Umfangs der Dienstvertretungsmacht nach der Arbeitsstellung nicht nur für den Vertretenen, sondern auch für den Vertragspartner eine große Gefahr. ${ }^{47}$ Denn für den Vertragspartner kann es sich als schwierig gestalten, nur aufgrund der Arbeitsstellung den Umfang der Dienstvertretungsmacht genau zu beurteilen, wenn die Grenzen zwischen der Dienstvertretungsmacht und der Arbeitsstellung unklar sind. Daher ist der zweiten Ansicht zuzustimmen und der Umfang von Prokura und Handlungsvollmacht durch die Rechtsprechung zu klären. Die Bestimmung des Umfangs der Dienstvertretungsmacht anhand ihres Typus (Prokura und Handlungsvollmacht) durch die Rechtsprechung wird die Anwendung des $\$ 170$ ZGB vereinfachen und verdeutlichen. Eine solche Bestimmung würde als Bekanntmachung des Umfangs der Dienstvertretungsmacht dienen, damit der Vertragspartner die inneren Verhältnisse zwischen dem Dienstvertreter und Vertretenen nicht zu prüfen braucht. Gleichzeitig könnten dadurch Handelsverkehrskosten gespart und die Verkehrseffizienz erhöht werden. ${ }^{48}$

\section{b) Umfang der Prokura und deren gesetzliche Beschränkung}

Eine Regelung über den Umfang der Prokura ist im ZGB nicht vorhanden. Es wird in der chinesischen Literatur vorgeschlagen, dass der Prokurist alle Arten von gerichtlichen und außergerichtlichen Geschäften außer

45 WANG Liming (Fn. 13), S. 640; RAN Keping/QU Yanni (Fn. 29), S. 64; ZHANG Xinbao (Fn. 13), S. 368.

46 YANG Qiuyu (Fn. 2), S. 102.

47 YIN Fei (Fn. 13), S. 60.

48 YIN Fei (Fn. 13), S. 55. 
der Veräußerung und Belastung von Grundstücken, Kreditvergabe oder Garantiegewähr an Dritten unternehmen kann. ${ }^{49}$ Dieser Ansicht ist zuzustimmen, da derjenige, der zu dem Prokuristen in Beziehung tritt, sich darauf verlassen muss, dass er so gestellt wird, als ob der Geschäftsherr ihm gegenüberstünde. ${ }^{50}$ Außerdem stellt das Betriebsgrundstück oftmals den bedeutendsten Vermögenswert des Unternehmens dar und der Unternehmensinhaber soll hinsichtlich der Veräußerung und Belastung von Grundstücken besonders geschützt werden. ${ }^{51}$ Ein Blick auf $₫ 49$ Abs. 1 HGB zeigt, dass dort der Prokurist ebenfalls Grundstücke des Kaufmanns nicht ohne eine besondere Vollmachtserteilung veräußern oder belasten darf.

Weiterhin wird vorgeschlagen, dass Grundlagengeschäfte ebenfalls nicht vom Prokuristen zu unternehmen sind wie etwa die Kapitalbeschaffung und -herabsetzung, ein Auflösungsbeschluss, die Bestellung von Abwicklern, eine Verschmelzung, eine Spaltung und ein Formwechsel sowie die Vermögensübertragung. ${ }^{52}$ Diese Ansicht ist vertretbar, denn die Grundlagengeschäfte sind aufgrund ihrer einschneidenden Wirkung für das Unternehmen so bedeutsam und gefährlich, dass sie nur vom Inhaber bzw. gesetzlichen Vertreter oder Mitglied des Vertretungsorgans wirksam getätigt werden dürfen. ${ }^{53}$

Darüber hinaus müssen die Geschäfte sich auf den Betrieb des Unternehmens beziehen. Privatgeschäfte und höchstpersönliche Geschäfte dienen nicht dem Betrieb des Unternehmens und sind deshalb nicht von der Prokura umfasst. ${ }^{54}$

Hinsichtlich einer Beschränkung des Umfangs der Prokura enthält das ZGB ebenfalls keine Regelungen. Einzig $\mathbb{\$} 148$ GG bestimmt, dass die Vorstandsmitglieder und leitenden Angestellten der Gesellschaft ohne die $\mathrm{Zu}$ stimmung der Hauptversammlung oder einen Vorstandsbeschluss keinen Kredit an Dritte vergeben, Dritten keine Garantie gewähren oder keine Insichgeschäfte unternehmen dürfen. Wenn der Prokurist zugleich ein Vorstandsmitglied oder ein leitender Angestellter der Gesellschaft ist, gilt die Bestimmung des $\$ 148$ GG auch für den Prokuristen. Wenn das Vorstandsmitglied oder der leitende Angestellte solche Geschäfte nicht unternehmen darf, gilt dies gleichermaßen auch für den einfachen Prokuristen.

49 GAN Peizhong (Fn. 27), S. 76; FAN Jian/JIANG Daxing (Fn. 27), S. 147.

50 Klaus Hofmann (Fn. 22), S. 14.

51 MüKoHGB/Krebs, 5. Auflage 2021, $₫ 49$ Rn. 36 f.

52 RAN Keping (Fn. 16), S. 142.

53 MüKoHGB/Krebs, 5. Auflage 2021, $₫ 49$ Rn. 5; Klaus Hofmann (Fn. 22), S. 69.

54 Klaus Hofmann (Fn. 22), S. 70. 


\section{Rechtsgeschäftliche Beschränkung}

Nach dem Grundsatz der Privatautonomie kann der Vollmachtgeber selbstverständlich den Umfang der Vollmacht bei der Vollmachterteilung ohne weiteres bestimmen und diese durch ein Rechtsgeschäft beschränken. Deshalb wird in der chinesischen Literatur vorgeschlagen, dass die rechtsgeschäftliche Beschränkung der Dienstvertretungsmacht durch eine Eintragung im Handelsregister möglich sei. ${ }^{55}$ Jedoch ist diese Ansicht fragwürdig, wenn die besonderen Erfordernisse der Sicherheit und Leichtigkeit des Handelsverkehrs berücksichtigt werden sollen. Im Folgenden sind die Möglichkeiten der rechtsgeschäftlichen Beschränkung der Prokura und Handlungsvollmacht jeweils aus rechtsvergleichender Sicht zu betrachten.

\section{a) Rechtsgeschäftliche Beschränkung der Prokura}

Im HGB ist die Regelung über den Umfang der Prokura eine zwingende gesetzliche Regelung. ${ }^{56} \mathrm{Nach} \ 50$ Abs. 1 HGB ist eine rechtsgeschäftliche Beschränkung der Prokura nicht möglich, sodass Wirksamkeit sowie Umfang der Prokura dadurch nicht beeinträchtigt werden können. ${ }^{57}$ Auch ist die Eintragung einer Beschränkung im Handelsregister Dritten gegenüber grundsätzlich unwirksam, es sei denn, es liegt ein Missbrauch der Vertretungsmacht vor. ${ }^{58}$ Diese Regelung dient der Sicherheit und Leichtigkeit des Rechtsverkehrs und betont die Trennung zwischen Vertretung und zugrunde liegendem Rechtsverhältnis, welches frei regelbar bleibt. ${ }^{59}$ Die Erfahrung und Erkenntnisse des deutschen Rechts können vom chinesischen Recht rezipiert werden.

\section{b) Rechtsgeschäftliche Beschränkung der Handlungsvollmacht}

Anders als bei der Prokura ist der Umfang der Handlungsvollmacht im deutschen Recht zwar gesetzlich bestimmt, aber abdingbar. ${ }^{60}$ Die Handlungsvollmacht kann zwar durch Rechtsgeschäft beschränkt werden, je-

55 YIN Fei (Fn. 13), S. 64.

56 MüKoHGB/Krebs, 5. Auflage 2021, \54 Rn. 5.

57 MüKoHGB/Krebs, 5. Auflage 2021, \50 Rn. 1.

58 MüKoHGB/Krebs, 5. Auflage 2021, $\$ 50$ Rn. 6.

59 MüKoHGB/Krebs, 5. Auflage 2021, \50 Rn. 1.

60 MüKoHGB/Krebs, 5. Auflage 2021, \54 Rn. 5. 
doch gelten solche Beschränkungen nach $₫ 54$ Abs. 3 HGB nur dann einem Dritten gegenüber, wenn er sie kennt oder kennen muss. Eine ähnliche Vorschrift findet sich auch in $\$ 170$ Abs. 2 ZGB, sodass im chinesischen Recht genauso eine Möglichkeit bestehen sollte, die Handlungsvollmacht durch Rechtsgeschäft beschränken zu können. Hinsichtlich des „Kennenmüssens" ist nach der h.L. im deutschen Recht für den gesamten Gutglaubensschutz im kaufmännischen Vertretungsrecht der Maßstab der groben Fahrlässigkeit bei der Beurteilung des Kennenmüssens des Dritten zu übernehmen, weil den Geschäftspartner keine allgemeine Nachforschungspflicht trifft. ${ }^{61}$ Den Geschäftspartner sollte daher in China ebenso zugunsten der Sicherheit und Leichtigkeit des Handelsverkehrs keine allgemeine Nachforschungspflicht treffen, es sei denn, der Vertragspartner verdient keinen Schutz.

\section{Die Beschränkung durch die zugrunde liegenden Verhältnisse}

Die Beschränkung der Dienstvertretung durch einen Arbeitsvertrag oder andere innere Bestimmungen ist eine Beschränkung im Innenverhältnis, ähnlich wie die Beschränkung des Bevollmächtigten durch den Auftrag im Fall der Vollmacht. Der Dienstvertreter ist nach den inneren Beschränkungen verpflichtet, bestimmte Regelungen, Abläufe oder Anweisungen des Vertretenen zu befolgen, etwa nur Rechtsgeschäfte zu unternehmen, deren Wert unter einer Million RMB liegen. Solche inneren Beschränkungen sind den Dritten nicht ersichtlich. Nach dem Gesetz unterliegt der Dritte keiner Nachforschungspflicht. ${ }^{62}$ Der Verkehrssicherheit halber sollen solche inneren Beschränkungen keine Wirkung nach außen entfalten. Das Abstraktionsprinzip der Vollmacht gilt auch für die Dienstvertretung, weil die Rechtsklarheit und Schnelligkeit des Handelsverkehrs bei der Handelsvertretung besonders wichtig sind. Der Vertragspartner kann dadurch auf die Dienstvertretungsmacht vertrauen und braucht nicht die inneren Verhältnisse zwischen dem Dienstvertreter und dem Vertretenen zu erforschen. Beispielsweise stellt nach der chinesischen Rechtsprechung der Ablauf des Verkaufs einer Wohnung eine innere Bestimmung dar, sodass der

61 MüKoHGB/Krebs, 5. Auflage 2021, \54 Rn. 44.

62 YANG Qiuyu (Fn. 2), S. 108; RAN Keping (Fn. 16), S. 148. 
Geschäftspartner zur Nachforschung einer solchen inneren Bestimmung nicht verpflichtet ist. ${ }^{63}$

In der chinesischen Literatur wird das Abstraktionsprinzip der Vollmacht von der h.M. zum Teil abgelehnt, weil der arglistige Vertragspartner dadurch auch geschützt und das Interesse des Vertretenen geschädigt wird. ${ }^{64}$ Danach sollte das die innere Beschränkung überschreitende Vertretergeschäft dem Vertretenen gegenüber wirksam sein, auch wenn der Vertragspartner diese Überschreitung kennt oder kennen müsste. Dieser Ansicht ist nicht zu folgen, weil dieses Problem durch das Rechtsinstitut des Missbrauchs der Vertretungsmacht beseitigt werden kann. Der Missbrauch der Vertretungsmacht ist von der deutschen Rechtsprechung zur Überwindung des Mangels des Abstraktionsprinzips der Vollmacht entwickelt worden. Danach gilt das Abstraktionsprinzip der Vollmacht dann nicht, wenn der Vertragspartner den Missbrauch kennt oder er so offensichtlich ist, dass er ihn hätte kennen müssen. ${ }^{65}$ Daher dient der Missbrauch der Vertretungsmacht als Grenze des Abstraktionsprinzips der Vollmacht. ${ }^{66}$ Eine ähnliche Auffassung wird auch noch für den Fall vertreten, dass der Dienstvertreter angesichts der Art des Geschäfts, des Betrags oder der Handelsgewohnheiten offensichtlich die Vertretungsmacht missbraucht und der Vertragspartner berechtigte Bedenken hat. Dann sei der Vertragspartner zur Nachforschung der möglichen inneren Beschränkung verpflichtet. ${ }^{67}$

Bei den inneren Verhältnissen stehen meistens Geschäftsgeheimnisse des Vertretenen auf dem Spiel, die nicht offenbart werden dürfen. Daher ist der Vertragspartner grundsätzlich nicht zu Nachforschungen solcher inneren Verhältnisse verpflichtet. Nur wenn der Vertragspartner die Überschreitung der inneren Verhältnisse des Dienstvertreters kennt oder wegen ihrer Offensichtlichkeit kennen müsste, liegt ein Missbrauch der Vertretungsmacht vor und der Vertretene braucht das vom Vertreter unternommene Rechtsgeschäft nicht gegen sich gelten zu lassen. Daher dient

63 Zivilurteil des Mittleren Volksgerichts der Stadt Zhunyi, Provinz Guizhou (2020) Qian 03 Min Zhong 2886.

64 RAN Keping (Fn. 16), S. 145; YE Jinqiang (叶金强), Zur Kausalität der Vollmachtserteilung (论代理权授予行为的有因构造), Tribune of Political Science and Law (政法论坛) 2010, Nr. 1, S. 112.

65 MüKoHGB/Krebs, 5. Auflage 2021, \50 Rn. 16.

66 CHI Ying (迟颖), Theorie des Missbrauchs der Vertretungsmacht im deutschen Recht und sein Referenzwert zum chinesischen Vertretungsrecht (德国法上的禁 止代理权滥用理论及其对我代理法的启示), Hebei Law Science (河北法学) 2020, Nr. 11, S. 64 ff.

67 RAN Keping (Fn. 16), S. 150. 
die Zusammenwirkung des Abstraktionsprinzips der Vollmacht und des Missbrauchs der Vertretungsmacht dazu, nicht nur den Handelsverkehr, sondern auch die Interessen des Vertretenen zu schützen.

\section{Rechtsfolge der Überschreitung der Dienstvertretungsmacht oder der inneren Beschränkung}

Nach $₫ 170$ Abs. 2 ZGB hat die Beschränkung des Umfangs der Dienstvertretungsmacht gegenüber gutgläubigen Dritten keine Wirkung. Diese Regelung macht keinen Unterschied zwischen gesetzlichen Beschränkungen, rechtsgeschäftlichen Beschränkungen oder inneren Beschränkungen. Darüber hinaus sind in $\$ 170$ Abs. 2 ZGB die Rechtsfolgen der Überschreitung der Beschränkung bei einem bösgläubigen Vertragspartner nicht statuiert. Diese Mängel des $₫ 170$ Abs. 2 ZGB haben dazu geführt, dass die Gerichte keiner konkreten Regelung folgen können und daher unterschiedliche Entscheidungen für ähnliche Fälle gefällt wurden. Deshalb ist es notwendig, die Rechtsfolgen bei der Überschreitung der Dienstvertretungsmacht im Wege der Auslegung der gesetzlichen Regelung des $\mathbb{1 7 0}$ Abs. 2 ZGB zu verdeutlichen.

\section{Rechtswirkung des Rechtsgeschäfts bei der Überschreitung der gesetzlichen Beschränkung der Dienstvertretungsmacht}

Über die Rechtswirkung des Rechtsgeschäfts bei der Überschreitung der gesetzlichen Beschränkung der Dienstvertretungsmacht wird gestritten. Auf der einen Seite wird vertreten, dass es für die Wirksamkeit eines solchen Rechtsgeschäfts auf die Gutgläubigkeit des Vertragspartners ankommt. ${ }^{68}$ Nach dieser Ansicht ist $\$ 170$ Abs. 2 ZGB auch auf den Fall der Überschreitung der gesetzlichen Beschränkung der Dienstvertretungsmacht anzuwenden. Auf der anderen Seite wird die Auffassung vertreten, dass das die gesetzliche Beschränkung überschreitende Rechtsgeschäft als Vertretung ohne Vertretungsmacht gilt und es nicht auf die Gutgläubigkeit des Vertragspartners ankommt. ${ }^{69}$ Daher findet nach dieser Auffassung $\$ 170$ Abs. 2 ZGB im Fall der Überschreitung der gesetzlichen Beschränkung der Dienstvertretungsmacht keine Anwendung. Der zweiten Auffas-

68 RAN Keping (Fn. 16), S. 143.

69 WANG Xiaohua (Fn. 17), S. 116. 
sung ist zuzustimmen. Der Vertragspartner muss die gesetzliche Beschränkung der Dienstvertretungsmacht kennen und ist verpflichtet, sich nach dem Vorhandensein der Dienstvertretungsmacht im Fall der möglichen Überschreitung der gesetzlichen Beschränkung der Dienstvertretungsmacht zu erkundigen. ${ }^{70}$ Wenn er dies nicht tut, darf er sich nicht auf $\$ 170$ Abs. 2 ZGB berufen und das Rechtsgeschäft dem Vertretenen gegenüber geltend machen. Vielmehr ist hier die Regelung über die Vertretung ohne Vertretungsmacht $(\mathbb{S} 171 \mathrm{ZGB})$ anzuwenden. Im deutschen Recht gilt das von dem Prokuristen unternommene und die gesetzlichen Beschränkungen überschreitende Rechtsgeschäft auch als Vertretung ohne Vertretungsmacht und $\$ 179$ BGB ist anzuwenden. ${ }^{71}$

Wenn der Prokurist ohne Erteilung einer besonderen Prokura Grundstücke veräußert oder belastet, an Dritte Kredite vergibt oder Garantien gewährt oder Grundlagenschäfte, Privatgeschäfte, höchstpersönliche Geschäfte oder ein Insichgeschäft unternimmt, gelten solche Rechtsgeschäfte als Vertretung ohne Vertretungsmacht. Zusätzlich zu den obengenannten Rechtsgeschäften sind die vom Handlungsbevollmächtigten unternommenen Rechtsgeschäfte zur Aufnahme von Darlehen oder zum Eingehen von Wechselverbindlichkeiten auch als Vertretung ohne Vertretungsmacht zu behandeln.

\section{Rechtswirkung des Rechtsgeschäfts bei der Überschreitung der} rechtsgeschäftlichen Beschränkung der Dienstvertretungsmacht und der inneren Beschränkung

\section{a) Wirksamkeit}

$\$ 170$ Abs. 2 ZGB bestimmt, dass die Beschränkung des Umfangs der Dienstvertretungsmacht dem gutgläubigen Vertragspartner gegenüber keine Wirkung entfaltet. Es wird vertreten, dass dies eine den Verkehr und den gutgläubigen Dritten schützende Sonderregelung der Anscheinsvollmacht darstellt. ${ }^{72}$ Die Anscheinsvollmacht ist eine Unterart der Vertretung ohne Vertretungsmacht. Wenn der Vertragspartner seine Gutgläubigkeit nicht beweisen kann, ist das Rechtsgeschäft ohne die Genehmigung des Vertretenen schwebend unwirksam. Dieser Ansicht ist nicht beizutreten,

70 RAN Keping/QU Yanni (Fn. 29), S. 71.

71 MüKoHGB/Krebs, 5. Auflage 2021, \49 Rn. 64; Klaus Hofmann (Fn. 22), S. 99.

72 RAN Keping/QU Yanni (Fn. 29), S. 70. 
da die Verkehrssicherheit dadurch nicht genügend geschützt wird. Die Regelung, dass die innere Beschränkung keine Wirkung nach außen hat, zielt im Gegenteil darauf ab, die Verkehrssicherheit zu schützen, und ist daher anders als die Anscheinsvollmacht. ${ }^{73}$ Vielmehr unterliegt $\$ 170$ Abs. 2 ZGB dem Grundsatz der Trennung von Vollmacht und zugrundliegendem Rechtsverhältnis. ${ }^{74}$ Dieser Ansicht ist zwar zuzustimmen, aber nur der Trennungsgrundsatz allein ist für den Verkehrsschutz nicht ausreichend, sondern es ist zusätzlich das Abstraktionsprinzip für die Auslegung des $\$ 170$ Abs. 2 ZGB anzuwenden. Danach ist das die innere Beschränkung überschreitende Rechtsgeschäft grundsätzlich wirksam und der Vertragspartner braucht nicht seine Gutgläubigkeit zu beweisen.

Wie oben schon erläutert, ist die Dienstvertretungsmacht (Prokura und Handlungsvollmacht) nicht rechtsgeschäftlich zu beschränken:

Eine rechtsgeschäftliche Beschränkung der Prokura hat grundsätzlich keine Wirkung nach außen mit der Folge, dass ein die rechtsgeschäftliche Beschränkung überschreitendes Rechtsgeschäft grundsätzlich wirksam ist, es sei denn, es handelt sich um einen Fall des Missbrauches der Vertretungsmacht. Eine rechtsgeschäftliche Beschränkung der Handlungsvollmacht wirkt nur dem Vertragspartner gegenüber, wenn er die Beschränkung kennt oder kennen muss.

Auch ist ein die rechtsgeschäftliche Beschränkung der Handlungsvollmacht überschreitendes Rechtsgeschäft wirksam. Nach dem Abstraktionsprinzip der Vollmacht hat die innere Beschränkung des Dienstvertreters grundsätzlich keine Wirkung auf den Umfang der Dienstvertretungsmacht. Nur wenn die Überschreitung der inneren Beschränkung als Missbrauch der Vertretungsmacht gilt oder der Vertragspartner die Beschränkung kennt oder kennen muss, ist die Wirksamkeit des die innere Beschränkung überschreitenden Rechtsgeschäfts fragwürdig. Entsprechend ist $\$ 170$ Abs. 2 ZGB nach dem Abstraktionsprinzip der Vollmacht erweiternd auszulegen, da mit „Beschränkung der Dienstvertretungsmacht“ in $\$ 170$ Abs. 2 ZGB nicht nur die rechtsgeschäftliche Beschränkung der Dienstvertretungsmacht, sondern auch die innere Beschränkung gemeint ist.

73 ZHU Guangxin (朱广新), Das Vertrauensschutzprinzip und seine Konstitution im Zivilrecht (信赖保护原则及其在民法中的构造), Beijing 2013, S. 158.

74 CHEN Su (Fn. 35), S. 1207. 


\section{b) Schwebende Unwirksamkeit}

Es wird in der chinesischen Literatur allgemein vertreten, dass ein die Dienstvertretungsmacht überschreitendes Rechtsgeschäft grundsätzlich als Vertretung ohne Vertretungsmacht gilt, wenn der Vertragspartner nicht gutgläubig ist. ${ }^{75}$ Dieser h.M. ist nicht zu folgen. Vielmehr ist „der gutgläubige Vertragspartner“ i.S.d. $₫ 170$ Abs. 2 ZGB einschränkend auszulegen, weil diese Regelung die Wirksamkeit des Rechtsgeschäfts im Fall der Überschreitung der rechtsgeschäftlichen Beschränkung der Prokura oder der inneren Beschränkung unangemessen einschränkt. In der Rechtsprechung ${ }^{76}$ und in der Literatur ${ }^{77}$ wird vielfach versucht, $\mathbb{\$} 170 \mathrm{Abs}$. 2 ZGB nur bei positiver „Kenntnis“ von der Überschreitung durch den Vertragspartner anzuwenden.

Dies kann auch für den Fall der Überschreitung der rechtsgeschäftlichen Beschränkung bei der Handlungsvollmacht gelten. Das vom Handlungsbevollmächtigten unternommene und die rechtsgeschäftlichen Beschränkungen überschreitende Rechtsgeschäft ist danach schwebend unwirksam, wenn der Vertragspartner die Beschränkung kennt oder aus grober Fahrlässigkeit nicht kennt.

Für den Fall der Überschreitung der rechtsgeschäftlichen Beschränkung der Prokura oder der Überschreitung der inneren Beschränkung soll $\$ 170$ Abs. 2 ZGB eng ausgelegt werden. Entsprechend ist vorzuschlagen, dass der Anwendung des Missbrauchs der Vertretungsmacht Vorrang gewährt werden muss gegenüber dem Schutz des Vertragspartners als gutgläubigen Dritten, weil die Tatbestandsmerkmale des Missbrauchs der Vertretungsmacht im Vergleich zur "Gutgläubigkeit des Vertragspartners“ viel strenger sind. Nur wenn der Prokurist die rechtsgeschäftliche Beschränkung der Dienstvertretungsmacht oder der Prokura oder wenn der Handlungsbevollmächtigte die innere Beschränkung vorsätzlich überschreitet und der Vertragspartner die Überschreitung kennt oder die Überschreitung so offensichtlich ist, dass der Vertragspartner sie kennen muss, gilt das von einem solchen Dienstvertreter unternommene Rechtsgeschäft als eine Ver-

75 CHEN Su (Fn. 35), S. 1211; WANG Liming (Fn. 13), S. 640; LI Shishi (Fn. 13), S. 528.

76 Zivilurteil des Mittleren Volksgerichts der Stadt Quzhou, Provinz Zhejiang (2020) Zhe 08 Min Zhong 785; Zivilurteil des Mittleren Volksgerichts der Stadt Dali Baizu Zizhizhou, Provinz Yunnan (2020) Yun 29 Min Zhong 18; Zivilurteil des Mittleren Volksgerichts der Stadt Deyang, Provinz Sichuan (2020) Chuan 06 Min Zhong 1059.

77 CHEN Su (Fn. 35), S. 1208. 
tretung ohne Vertretungsmacht. ${ }^{78}$ Somit ist ein von dem Prokuristen unternommenes und die rechtsgeschäftlichen Beschränkungen überschreitendes Rechtsgeschäft oder ein von dem Prokuristen und dem Handlungsbevollmächtigten unternommenes und die inneren Beschränkungen überschreitendes Rechtsgeschäft nur ausnahmsweise als Vertretung ohne Vertretungsmacht einzustufen. Im Fall des Missbrauchs der Vertretungsmacht ist das Rechtsgeschäft wie die Vertretung ohne Vertretungsmacht schwebend unwirksam, kann aber durch die Genehmigung des Vertretenen zur endgültigen Wirksamkeit gelangen.

Mit der obigen Auslegung des $\$ 170$ Abs. 2 ZGB ist ein vom Prokuristen unternommenes und die rechtsgeschäftlichen Beschränkungen überschreitendes Rechtsgeschäft oder ein vom Prokuristen und Handlungsbevollmächtigten unternommenes und die inneren Beschränkungen überschreitendes Rechtsgeschäft grundsätzlich wirksam, es sei denn, es liegt ein Missbrauch der Vertretungsmacht vor. Im Fall des Missbrauchs der Vertretungsmacht gilt das Rechtsgeschäft als Vertretung ohne Vertretungsmacht i. S. d. $\$ 171$ ZGB. Danach kann der Vertretene es genehmigen.

3. Haftung bei der Überschreitung der Dienstvertretungsmacht oder der inneren Beschränkung

\section{a) Haftung des Dienstvertreters bei Wirksamkeit des Rechtsgeschäfts}

Wenn das die Dienstvertretungsmacht oder die innere Beschränkung überschreitende Rechtsgeschäft wirksam ist, ist der Vertretene Vertragspartei und zur Erfüllung des Rechtsgeschäfts verpflichtet. Der Dienstvertreter haftet nicht dem Vertragspartner gegenüber. Jedoch haftet der Dienstvertreter dem Vertretenen gegenüber aufgrund seines Arbeitsverhältnisses bzw. durch die gesetzlichen Regelungen des Auftrags ( $\$ 929$ Abs. 2 ZGB) auf Schadenersatz wegen Nichterfüllung der inneren Pflicht. Darüber hinaus kann der Vertretene dem Dienstvertreter auf Grundlage des Arbeitsvertrags oder aus arbeitsrechtlichen Gründen kündigen.

78 CHI Ying (Fn. 66), S. 64 ff. 


\section{b) Haftung des Dienstvertreters bei Unwirksamkeit des Rechtsgeschäfts}

$\$ 171$ Abs. 3 ZGB bestimmt, dass der Vertreter ohne Vertretungsmacht nach Wahl des gutgläubigen Vertragspartners entweder den Vertrag erfüllen oder Schadensersatz leisten soll. Danach hat nur der gutgläubige Vertragspartner einen Anspruch auf Schadensersatz wegen Vertretung ohne Vertretungsmacht. Dagegen bestimmt $\mathbb{} 171$ Abs. 4 ZGB, dass der Dienstvertreter dem Vertragspartner gegenüber nach seinem Verschulden zu Schadensersatz verpflichtet ist, auch wenn der Vertragspartner nicht gutgläubig ist. Danach kann der Dienstvertreter von der Haftung für eine Vertretung ohne Vertretungsmacht nicht wegen einer fehlenden Gutgläubigkeit des Vertragspartners entlassen werden. Die Regelung der $\$ 171$ Abs. 4 ZGB ist aber fragwürdig, weil sie in Widerspruch zum $₫ 171 \mathrm{Abs.} 3$ ZGB steht. Denn die Haftung des Vertreters dient bei der Vertretung ohne Vertretungsmacht dem Schutz des gutgläubigen Vertragspartners. Wenn der Vertragspartner nicht gutgläubig ist, verdient er keinen Schutz. Im deutschen Recht haftet der Vertreter auch nicht, wenn der Vertragspartner nicht gutgläubig ist. ${ }^{79}$ Kennt der Vertragspartner die Überschreitung der Dienstvertretungsmacht und den Missbrauch der Vertretungsmacht oder sind diese so offensichtlich, dass der Vertragspartner sie kennen muss, haftet der Dienstvertreter dem Vertragspartner gegenüber nicht.

\section{Zusammenfassung}

Die Dienstvertretung ist eine rechtsgeschäftliche Handelsvertretung, bei der ein Mitarbeiter (kein gesetzlicher Repräsentant) des Vertretenen als Dienstvertreter in dessen Namen ein für oder gegen ihn geltendes Rechtsgeschäft unternimmt. Sie unterscheidet sich von dem gesetzlichen Repräsentanten und der Vollmacht. Die Dienstvertretungsmacht im chinesischen Recht ist nach deutschem Vorbild durch Gesetzesauslegung als Prokura und Handlungsvollmacht zu typisieren. Mit solch einer Typisierung ist der Umfang der Dienstvertretungsmacht gesetzlich festgelegt und dessen Beschränkung offenkundig. Die Typisierung der Dienstvertretungsmacht dient vor allem der Sicherheit und Einfachheit des Handelsverkehrs, aber auch den Interessen des Vertretenen und Vertreters. Der Vertragspartner braucht sich nicht um die Vertretungsmacht des Dienst-

79 Karl Larenz/Manfred Wolf, Allgemeiner Teil des Bürgerlichen Rechts, 9. Auflage, München 2004, S. 909. 
vertreters beim Geschäftsherrn zu vergewissern und der Dienstvertreter braucht nicht zu befürchten, dass er als Vertreter ohne Vertretungsmacht handelt.

Der vom Gesetz festgelegte Umfang der Prokura und der Handlungsvollmacht ist nicht zu überschreiten. Das vom Dienstvertreter unternommene, den gesetzlichen Umfang überschreitende Rechtsgeschäft gilt als Vertretung ohne Vertretungsmacht i. S. v. $\$ 171$ ZGB. Die Regelung über die Anscheinsvollmacht ( $\$ 170$ Abs. 2 ZGB) findet hier keine Anwendung. Dagegen ist ein die rechtsgeschäftliche Beschränkung oder die innere Beschränkung überschreitendes Rechtsgeschäft nach $\$ 170$ Abs. 1 ZGB grundsätzlich wirksam, es sei denn, es handelt sich um einen Fall des $₫ 170$ Abs. 2 ZGB.

Wenn das die Dienstvertretungsmacht oder die innere Beschränkung überschreitende Rechtsgeschäft wirksam ist, haftet der Dienstvertreter nicht dem Vertragspartner gegenüber. Jedoch kann er wegen Verletzung seiner Pflichten aus dem Arbeitsvertrag oder wegen Verletzung der Vorschriften des Auftragsrechts ( $\$ 929$ Abs. 2 ZGB) auf Schadenersatz gegenüber dem Geschäftsherrn haften. Wenn das die Dienstvertretungsmacht oder die innere Beschränkung überschreitende Rechtsgeschäft nichtig ist, haftet der Dienstvertreter dem Vertragspartner gegenüber auch dann nicht, wenn der Vertragspartner die Überschreitung der Dienstvertretungsmacht kennt oder sie bei deren Offensichtlichkeit kennen muss. 
